\title{
Methodology for measuring the gap size using a fiber-optic displacement sensor exemplified by a centrifugal blood pump
}

\author{
Maciej Gawlikowski, ${ }^{* 1,2}$ Przemysław Kurtyka, ${ }^{1,2}$ Jerzy Zalewski, ${ }^{1}$ Magda Zarwańska-Doffek, ${ }^{1}$ Artur Kapis ${ }^{1}$ \\ ${ }^{1}$ Foundation of Cardiac Surgery Development, Wolności 345a, 41-800 Zabrze, Poland \\ ${ }^{2}$ Faculty of Biomedical Engineering, Silesian University of Technology, Roosevelta 40, 41-800 Zabrze, Poland
}

Received May 14, 2020; accepted June 21, 2020; published June 30, 2020

\begin{abstract}
In order to avoid blood clotting, in the second generation of rotary blood pumps the impeller is suspended without mechanical bearing, using the balance of magnetic and hydrodynamic forces. Reaching single tens of microns, the gap between the pump housing and the impeller is crucial for the level of blood traumatization by the pump. In this paper we would like to present the method of physical measurement of this gap on a running pump with the use of a commercial fiber-optic proximity sensor exemplified by the Polish rotary blood pump ReligaHeart ROT. We have also discussed the technical requirements of laboratory stand construction.
\end{abstract}

Since the beginning of the XXI century the implantable rotary blood pumps (IRBP) have been used for treatment of patients suffering from end stage heart insufficiency [1]. In $1^{\text {st }}$ generation of axial IRBP (Heart Mate II / Thoratec) the impeller was suspended on sapphire mechanical bearings, which resulted in blood clotting and caused blood trauma [2]. A breakthrough in IRBP construction was developing the method of bearingless impeller suspension. In $2^{\text {nd }}$ generation of centrifugal IRBP (HVAD / Heartware-Medtronic), rotor levitation is achieved by the balance of forces of different nature, acting on the impeller: magnetic (coming from the permanent magnets and motor winding) as well as hydrodynamic (coming from the pumping liquid and from special blades of a hydraulic bearing, located on the surface of impeller) [3]. In $3^{\text {rd }}$ generation of IRBP (Heart Mate III / Abbott), rotor levitation is assured by an active automation system and occurs even when the impeller is not rotating [4].

The rotor bearingless suspension results in a gap between the impeller and pump housing, which is filled with blood. The blood cells (erythrocytes, thrombocytes and leucocytes) as well as coagulation proteins (fibrinogen, von Willebrand protein etc.) are subjected to shear stress, the greater it becomes, the smaller the gap is. Excessive shear stress affecting the blood causes its significant traumatization: damage of red blood cells (haemolysis), activation of platelets leading to the activation of a coagulation system (thrombogenicity) and segmentation of von Willebrand protein (resulting in gastrointestinal bleeding) [5]. Therefore, the IRBP manufacturers strive to make the gap as big as possible

*E-mail: maciej.gawlikowski@ polsl.pl while maintaining excellent hydrodynamic features of the pump.

In $2^{\text {nd }}$ generation of IRBP, the gap size may be measured only during impeller rotation, because only then a lifting hydrodynamic force is generated. For this reason researchers and constructors assess the impeller levitation height only by computer fluid dynamic (CFD) simulations. However, numerical simulation of a blood flow is a complex problem [6]. In a first simplification, blood is a non-Newtonian liquid and except for the shear rate its viscosity depends on a haematocrit level and fibrinogen concentration. Next, it was clearly proven [6], that blood rheology and the nature of its flow through narrow gaps depends on the flexibility of red blood cells membranes and its susceptibility to cell shape deformation. Finally, to carry out accurate results of CFD simulations, blood has to be regarded as a non-Newtonian suspension of deformable particles with micron sizes in Newtonian plasma. Finally, the problem of CFD simulations of IRBP is mesh creation in a narrow gap. Because of the reasons listed above, the physical measurement of impeller levitation height plays an important role in IRBP designing and prototypes testing.

The aim of the present work was to develop a physical method of measuring the size of the gap between the pump housing and the impeller on a running pump.

A measurement system was developed for Polish, centrifugal IRBP ReligaHeart ROT (Foundation of Cardiac Surgery Development, Zabrze, Poland) with a passively suspended impeller (Fig. 1) [7].

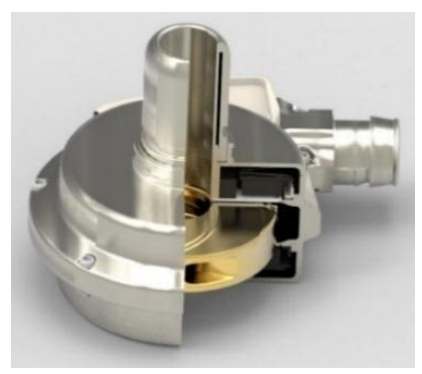

Fig. 1. Implantable rotary blood pump ReligaHeart ROT.

In this pump the bearingless rotor suspension is assured by permanent magnets and a hydrodynamic bearing located at the upper and lower surfaces of the impeller. A 
cross section of the pump with the main elements marked is shown in Fig. 2.

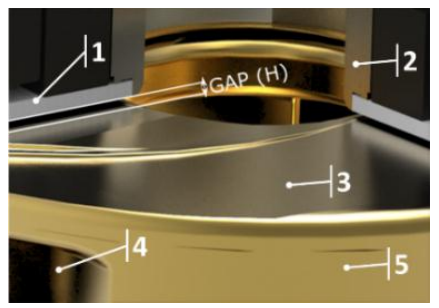

Fig. 2. Cross section of the ReligaHeart ROT with a marked gap: (1) pump housing, (2) inflow cannula, (3) blade of upper hydrodynamic bearing, (4) flow canal, (5) impeller.

The internal surfaces of the pump and each component part of the impeller was covered by the TiN layer which assures low thrombogenicity [8]. A two-section, sensorless BLDC motor can generate $27 \mathrm{mNm}$ of torque.

The CFD simulations and magnetic as well as hydrodynamic forces measurement revealed, that under standard operation conditions (3000RPM, 100mmHg of load pressure and $3.5 \mathrm{cP}$ of liquid viscosity) the height of impeller levitation should be ap. $50 \mu \mathrm{m}$. However, during in-vitro experiments on blood, high haemolysis $(\Delta \mathrm{fHB}=2 \mathrm{~g} / 1$ after 5 hours of circulation) was observed, which indicated a smaller levitation height than calculated. It was a reason for seeking a physical method of measurement of impeller levitation height.

For the gap height measurement, we decided to use a commercial, fibre-optic, proximity sensor (RC-19/Philtec, USA) with reflectance compensation. According to the manufacturer's specifications, this sensor is intended for rotating machines and can operate in light transparent liquids. The main technical parameters of the sensor were summarized in Table 1. The key feature of the sensor is an extremely small tip $(0.8 \mathrm{~mm}$ in diameter, including metal leader) and customized tip construction (Fig. 3), which allowed the sensor to be led inside the pump between the motor winding.

Table 1. The technical parameters of RC-19 displacement sensor.

\begin{tabular}{|l|r||l|r|}
\hline Light source & LED, 850nm & Tip diameter & $0.8 \mathrm{~mm}$ \\
\hline $\begin{array}{l}\text { Light beam } \\
\text { spread }\end{array}$ & $60^{\circ}$ & $\begin{array}{l}\text { Resolution } \\
\text { @ 20kHZ }\end{array}$ & $0.30 \mu \mathrm{m}$ \\
\hline $\begin{array}{l}\text { Bandwidth } \\
(3 \mathrm{db})\end{array}$ & $200 \mathrm{kHz}$ & $\begin{array}{l}\text { Resolution } \\
@ 100 \mathrm{~Hz}\end{array}$ & $0.15 \mu \mathrm{m}$ \\
\hline Total range & $760 \mu \mathrm{m}$ & Output & analog \\
\hline $\begin{array}{l}\text { Near field } \\
\text { range }\end{array}$ & $150 \mu \mathrm{m}$ & Sensitivity & $9 \mathrm{mV} / \mu \mathrm{m}$ \\
\hline
\end{tabular}

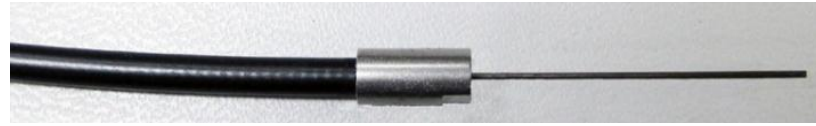

Fig. 3. RC-19 fibre-optic cable and customized sensor tip.

The most difficult technical problem was to drill a $0.8 \mathrm{~mm}$ diameter hole through the $0.6 \mathrm{~mm}$ thick zirconium plate which separates the interior of the pump having permanent contact with the blood from the chamber containing motor windings and magnetic core. The optical sensor tip was located between the motor winding and permanent magnets of the magnetic bearing (Fig. 4). To avoid operation in the near field, the sensor tip was moved $220 \mu \mathrm{m}$ back from the external surface of the zirconium plate by applying of a metal pad. That distance in relation to the hole diameter assured that a light beam was not shadowed by the edge of the hole (Fig 4, on the right).
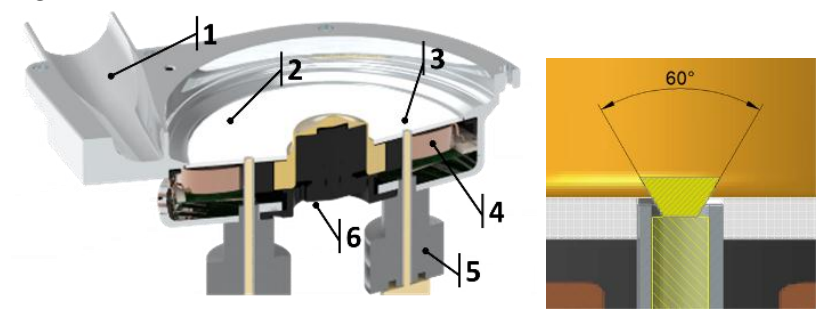

Fig. 4. (left) Fibre-optic sensor mounting method: (1) pump housing and outflow cannula, (2) zirconium plate, (3) sensor tip, (4) motor winding,

(5) sensor pilot, (6) bottom permanent magnetic bearing; (right) geometry of a light beam in relation to the sensor tip position showing the lack of beam shadowing by the edge of the hole.

According to the principles of operation of reflectance displacement sensors [9], the transfer function depends on the refractive index of a liquid in which the light wave propagates as well as the reflectance of a mirroring surface. In our case, the operation liquid was $40 \%$ glycerine in a water solution which mimics blood viscosity and its light transparency. During the pump manufacturing, the impeller surface is polished to achieve the roughness $R_{a}=0.08$ and next it is covered by a TiN layer in order to avoid blood clotting [8].

The transfer function of the system was determined under static conditions by gradual sample offsetting from the sensor tip. In order to maintain similar reflectance to that of the impeller, the sample was made in the same manner like the impeller (Ti6Al7Nb alloy, polishing to reach $R_{a}=0.08$ and covering by a TiN layer in an identical manufacturing process). The sample was mounted onto the reference electronic micrometre (1726 / HeliosPreisser) in such a way as to ensure parallelism with the zirconium plate and to avoid any backlashes. The accuracy of a reference micrometre was $1.0 \mu \mathrm{m}$. Next, the volume between the sample and sensor tip was filled with an peration liquid so as to eliminate any air bubbles. Measurements were carried out for distances from 0 to $460 \mu \mathrm{m}$ with a step of $20 \mu \mathrm{m}(\mathrm{n}=6$ measurements at each point were performed), where 0 means, that the sample lies on the zirconium plate without any backlash. The transfer function was determined in Statistica 13 by the $4^{\text {th }}$ order polynomial regression method (1). The regression coefficients and their confidence intervals were presented in Table 2. The standard error of estimation was $\varepsilon=0.2013 \mu \mathrm{m}$. The distribution of residues was normal (result of Shapiro-Wilk test $\mathrm{p}=0.3884$ )

$$
H=\sum_{i=0}^{4} a_{i} \cdot U^{i} \pm \epsilon
$$


where: $\mathrm{H}[\mu \mathrm{m}]$ - distance of the sample from the sensor tip, which means impeller levitation height, $U[\mathrm{mV}]$ - output sensor voltage, $\varepsilon$ $[\mu \mathrm{m}]-$ standard error of estimation.

Table 2. Regression coefficients and its statistical significance.

\begin{tabular}{|c|c|c|c|c|}
\hline $\mathbf{a}_{\mathbf{n}}$ & Value & $\begin{array}{c}\text { Lower } \\
\text { confidence } \\
\text { interval } \\
\end{array}$ & $\begin{array}{c}\text { Upper } \\
\text { confidence } \\
\text { interval }\end{array}$ & $\mathbf{p}$ \\
\hline $\mathbf{a}_{\mathbf{0}}$ & $-1.2437 \mathrm{e} 02$ & $-1.2846 \mathrm{e} 02$ & $-1.2029 \mathrm{e} 02$ & \multirow{5}{*}{ 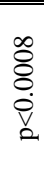 } \\
\hline $\mathbf{a}_{1}$ & $4.5036 \mathrm{e}-01$ & $4.3336 \mathrm{e}-01$ & $4.6736 \mathrm{e}-01$ & \\
\hline $\mathbf{a}_{2}$ & $-2.3820 \mathrm{e}-04$ & $-2.6082 \mathrm{e}-04$ & $-2.1558 \mathrm{e}-04$ & \\
\hline $\mathbf{a}_{3}$ & $9.5756 \mathrm{e}-08$ & $9.5756 \mathrm{e}-08$ & $9.5756 \mathrm{e}-08$ & \\
\hline $\mathbf{a}_{4}$ & $-1.4167 \mathrm{e}-11$ & $-1.4167 \mathrm{e}-11$ & $-1.4167 \mathrm{e}-11$ & \\
\hline
\end{tabular}

The assessment of measurement accuracy was carried out by comparing the depth of a hydrodynamic bearing blade measured by a fibre-optic system and measured (by coordinate machine 3D Contura / Zeiss, accuracy $1.2 \mu \mathrm{m}$ ) just after manufacturing the impeller. Optical measurements were carried out on the running pump under standard operation conditions. The idea of measurement was explained in Fig. 5: the position of the fibre-optic sensor relative to the hydrodynamic bearing blade was marked with a white, dashed line A-C. At the point $\mathrm{A}$, there is a recess with the depth $h \approx 160 \mu \mathrm{m}$ (dependending on manufacturing accuracy). In this point a hydrodynamic bearing blade begins. On the section A$\mathrm{B}$, the blade depth decreases and on the section $\mathrm{B}-\mathrm{C}$, the blade is flat. The cross section of a blade profile along the line A-C was drawn as a red line. The output voltage (measured by means of oscilloscope MSO 4054 / Agilent) and distance calculated from Eq. (1) were shown below.

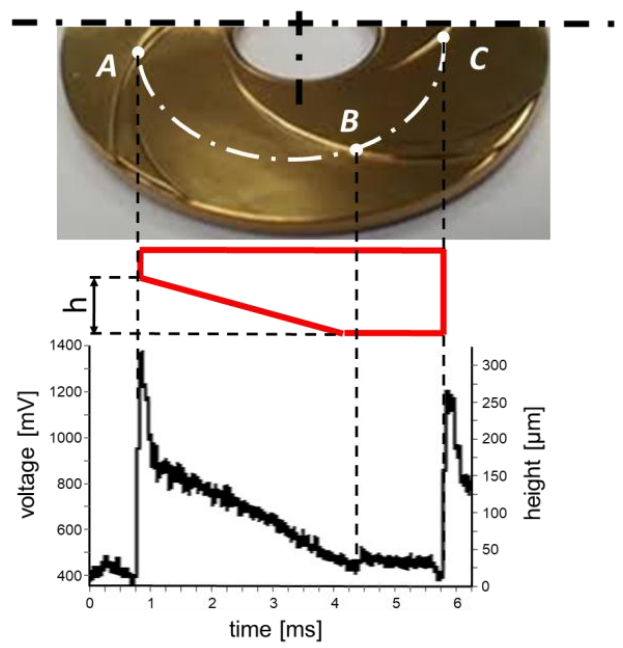

Fig. 5. View of a hydrodynamic bearing with a marked cross-section line (top); the profile of one blade of the hydrodynamic bearing; voltage and distance measured by a fibre-optic sensor.

The hydrodynamic bearing of the investigated pump consists of 4 blades. Each blade was measured $n=6$ times using the investigated fibre-optic system. The results of measurements were presented in Table 3. According to presented data the total absolute accuracy of the investigated fibre-optic system is $\pm 3.2 \mu \mathrm{m}$.
Table 3. Comparison of blade depth $(h)$ measurements using investigated fibre-optic sensor and reference coordinate machine: $\mathrm{M}$ mean value, C.I.-confidence interval.

\begin{tabular}{|c||c|c||c|c|}
\hline \multicolumn{1}{|c||}{} & \multicolumn{2}{c||}{ REFERENCE } & \multicolumn{2}{c|}{ FIBRE-OPTIC } \\
\cline { 2 - 5 } & $\mathrm{M}[\mu \mathrm{m}]$ & C.I. $[\mu \mathrm{m}]$ & $\mathrm{M}[\mu \mathrm{m}]$ & C.I. $[\mu \mathrm{m}]$ \\
\hline $\mathbf{1}$ & 161.20 & $160.00-162.40$ & 161.57 & $159.91-163.23$ \\
\hline $\mathbf{2}$ & 161.00 & $158.80-162.20$ & 161.57 & $159.77-163.37$ \\
\hline $\mathbf{3}$ & 187.80 & $186.60-189.00$ & 188.08 & $186.86-189.30$ \\
\hline $\mathbf{4}$ & 154.60 & $153.40-155.80$ & 154.11 & $152.81-155.41$ \\
\hline
\end{tabular}

The RC-19 sensor is intended for displacement and vibration measurement of rotating elements. It has been equipped with two groups of fibres in order to compensate for the reflectance changes from different surfaces [10]. In order to make reliable and accurate measurement certain requirements have to be met. The sensor cannot work in the near-field $(0 \div 150 \mu \mathrm{m})$ due to a non-monotonic transfer function in this region. Measurements may be carried out only on a light transparent liquid, so the use of the sensor on the blood may be difficult (however, for several microns gaps it probably will be possible). If the surface reflectance or operation liquid changes the in-situ calibration is indispensable. Any air bubbles in the path of the optical beam will disturb the measurements due to light reflection and light beam refraction in environments with different optical properties. The light beam cannot be shadowed by any edges or surfaces, so the mechanical project of mounting the sensor tip must consider this requirement. We also observed the influence of pump housing stiffness on the results of displacement measurements: the ap. $10 \mathrm{~N}$ of a longitudinal pressing force caused a $7 \mu \mathrm{m}$ change in the results. The voltage spikes visible in Fig. 5 at time $t=1 \mathrm{~ms}$ and $t=6 \mathrm{~ms}$ are caused by light refraction on a step change in the depth of a hydrodynamic bearing blade and it is typical of reflectance sensors [10].

The demonstrated fibre-optic system was applied to advanced investigation concerning the assessment of impeller levitation height in a Polish, centrifugal rotary blood pump ReligaHeart ROT.

This work has been financially supported by the Polish National Centre of Research and Development (RHROT/266798/STRATEGMED-II).

\section{References}

[1] S. Westaby, Future Cardiol. 9, 2 (2013).

[2] R. Delgado, M. Bergheim, Epert Rev. Med. Devices, 2, 5 (2005).

[3] M. Ozban, T. Yagdi, C. Engin et al, Transplant proc., 44, 6 (2012).

[4] A.T. Lanfear, M. Hamandi, J. Fan et al., J. Card. Surg., 35, 1 (2020).

[5] Ch. Zengsheng et al., Medic. Novel Techn. Dev. 3, 100024 (2019).

[6] A.M. Robertson et al., Rheological models of blood in: L. Formaggia, A. Quarteroni, A Veneziani (eds) Cardiovascular Mathematics (Milano, Springer-Verlag 2009).

[7] M. Gawlikowski et al., Adv. Intell. Systems Comput. 925 (2019).

[8] R. Kustosz, et al., Arch. Matall. Mater. 60, 3 (2015).

[9] S. S. Patil, A. D. Shaligram, IJSER, 4, 11 (2013).

[10] Philtec Application Note, 6, 25 (2017), https://philtec.com/wpcontent/uploads/2019/06/V6N25_ReflectanceCompensated.pdf 